\title{
Novel Approach for the Determination of the Taylor-Quinney Coefficient
}

\author{
Sebastian Härtel ${ }^{1, a^{*}}$, Marcel Graf ${ }^{1, b}$, Birgit Awiszus ${ }^{2, c}$, \\ Kevin Gordon Abstoss ${ }^{3, d}$ and Rafael Hild ${ }^{4, e}$ \\ ${ }^{1}$ Steinbeis Research Center Production and Material Technology, Chemnitz, Germany \\ ${ }^{2} \mathrm{TU}$ Chemnitz, Professorship Virtual Production Engineering, Chemnitz, Germany \\ ${ }^{3} \mathrm{TU}$ Chemnitz, Professorship of Welding Engineering, Chemnitz, Germany \\ ${ }^{4}$ RWTH Aachen, Chair of Manufacturing Technology, Aachen, Germany \\ a sebastian.haertel@stw.de, ${ }^{b}$ marcel.graf@stw.de, ${ }^{c}$ birgit.awiszus@mb.tu-chemnitz.de, \\ dkevin.abstoss@mb.tu-chemnitz.de, ${ }^{\mathrm{e}}$ r.hild@wzl.rwth-aachen.de
}

Keywords: Taylor-Quinney coefficient, compression test, adiabatic heating

\begin{abstract}
Within this study, a new method for the determination of the Taylor-Quinney coefficient is presented. The coefficient was identified by measuring the force-displacement-behavior as well as the temperature change resulting from an adiabatic compression test. In order to deduce the global temperature increasing of the specimen from the local measured temperature a suitable specimen geometry was designed with the use of numerical simulation. The resulting specimen allows a friction-free compression and therefore precludes a temperature increase through friction. Finally, the Taylor-Quinney coefficient of C35 steel (1.0501) was experimentally determined in the initial state as well as after a heat treatment.
\end{abstract}

\section{Introduction}

During forming processes, a large amount of the performed plastic work $\mathrm{W}_{\text {plast }}$ is converted directly into thermal energy Q, due to material dependent internal friction [1]. The Taylor-Quinney coefficient $\beta$ (Eq. 1) defines the percentage that is converted adiabatically into thermal energy [2].

$$
\beta=\frac{\mathrm{Q}}{\mathrm{W}_{\text {plast }}}=\frac{\mathrm{c}_{\mathrm{p}} \cdot \mathrm{m} \cdot \Delta \mathrm{T}}{\mathrm{W}_{\text {plast }}}
$$

Generally, in case of steel it can be assumed that ca. $90 \%$ of the plastic work are converted into heat [8]. The resulting change in temperature $\Delta \mathrm{T}$ can be calculated with Eq. 2 by assuming adiabatic conditions during forming. This means that the resulting heat cannot dissipate through heat conduction due to the short process time.

$$
\Delta \mathrm{T}=\frac{\beta}{\mathrm{c}_{\mathrm{p}} \cdot \mathrm{m}} \cdot \mathrm{W}_{\text {plast }}
$$

In order to calculate the temperature distribution or rather the heating of a workpiece realistically, it is essential to experimentally determine the Taylor-Quinney coefficient as precisely as possible (e.g. with numerical simulation of forming processes).

Within literature, numerous possible methods are described to determine the Taylor-Quinney coefficient. Investigations of [3] showed the examination of the Taylor-Quinney coefficient $\beta$ of pure magnesium and magnesium alloy with high strain rates $\left(\dot{\varphi} \geq 1000 \mathrm{~s}^{-1}\right)$ and the use of a SplitHopkinson-setup. Temperature was detected by using an infrared camera. Results show that the conversion factor is significantly below a general assumed value of 0.9 in case of both materials. Furthermore, the factor of the investigated magnesium materials shows a strong dependency on the load direction. A strain rate dependent behavior was not observed. Rosakis et al. [4] also investigated the Taylor-Quinney coefficient $\beta$ with the use of the Split-Hopkinson-setup. However, they investigated an aluminum alloy AA-2024 as well as $\alpha$-titanium. The results exhibit that the Taylor-Quinney coefficient $\beta$ of AA-2024 depends from the plastic strain but not from the strain rate. On the contrary, the Taylor-Quinney coefficient $\beta$ of $\alpha$-titanium did not show a dependency 
from the plastic strain but from the strain rate. Knysh und Korkolis [5] used a uniaxial tensile test as well as an infrared camera for determination of the Taylor-Quinney coefficient $\beta$ of stainless steel $1.4305,1.4401$ as well as titanium Ti-6Al-4V and pure titanium grade 5. The experiments were performed under vacuum atmosphere in order to control the thermal loss caused by convection. The results showed that the conversion factor decreases with increasing plastic strain. Fekete and Szekeres [6] quantified the influence of stress state on the Taylor-Quinney coefficient $\beta$. For that purpose, a special specimen geometry was developed to perform compression and tension tests on a thermo-mechanical forming simulator GLEEBLE 3800. The investigations showed that the TaylorQuinney coefficient $\beta$ of $15 \mathrm{Ch} 2 \mathrm{MFA}$ and $8 \mathrm{Ch} 18 \mathrm{~N} 10 \mathrm{~T}$ is lower during compression stresses than during tensile stresses.

The presented methods are disadvantageous with regards to analytical calculation of thermal loss through conduction, convection etc. during low strain rate conditions. This leads to inaccuracies during the determination of the Taylor-Quinney coefficient $\beta$ due to the simplified assumptions. Furthermore, the determination of the Taylor-Quinney coefficient $\beta$ with the use of compression tests is falsified because friction occurs between the front side of the specimen and the tools. Additionally, the measurement of temperature change with the use of infrared camera or thermocouples is based on the assumption that the local measured temperature change at the surface is equivalent to the average temperature change of the component. Especially during compression tests, the temperature increase inside the specimen is significantly higher than at the surface.

Based on the analyzed methods for determination of the Taylor-Quinney coefficient $\beta$, the present research is aiming on a method, which meets following requirements:

- simple and solid experimental setup

- realization of high plastic strain

- achieving high strain rates in order to guarantee adiabatic conditions

- almost frictionless deformation in order to neglect heating through friction at front side

- simple temperature detection with the help of thermocouples

\section{Approach}

In order to achieve high plastic strain the compression test was used. The tests were performed with the use of a thermo-mechanical testing system GLEEBLE 3800 because the high stroke speed of $2 \mathrm{~m} / \mathrm{s}$ guarantees an adiabatic deformation of the specimen.

A specific specimen geometry was developed aiming on a minimization of friction during the upsetting. A cylindrical specimen was tapered at the measuring area as shown in Fig. 1. With this geometric and monolithic design, a relative movement of the measuring area towards the forming tools could be successful avoided. Therefore, a temperature increase caused by friction could be excluded in this area. Additionally, the diameter of the supporting area is much larger than of the measuring area, which leads to the fact that deformation only takes place at the testing range. The transition radius between measuring area and support area of the specimen leads to a homogeneous stress distribution during testing. With methods of inverse analyses, the specimen was optimized with regards to global and local temperature changes. As a result, the change of temperature at a defined measuring point $\Delta \mathrm{T}_{\text {local }}$ is equal to the average temperature change of the whole specimen $\Delta \mathrm{T}_{\text {global. }}$. This optimizing step is necessary due to the formation of a temperature field caused by inhomogeneous distribution of forming degree during upsetting. 

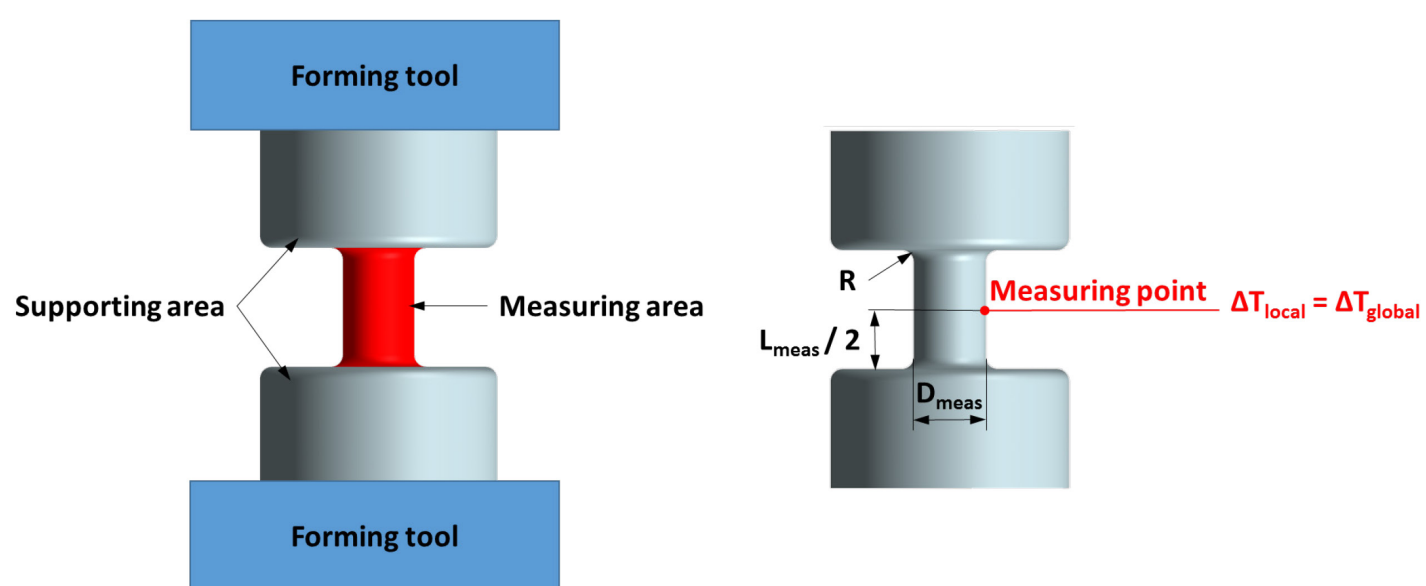

Fig. 1. Newly developed specimen geometry for the determination of Taylor-Quinney coefficient (schematically)

\section{Materials}

For the determination of the Taylor-Quinney coefficient $\beta$, a quenched and tempered steel C35 (1.0501) was used in initial state (as received) and after a defined heat treatment. The heat treatment consists of a 6 hour annealing at $1000{ }^{\circ} \mathrm{C}$ with a cooling rate of $1 \mathrm{~K} / \mathrm{s}$, leading to a change in microstructure, as shown in Fig. 2. The grain size increases from $25 \mu \mathrm{m}$ at the initial state to $75 \mu \mathrm{m}$ due to the heat treatment.
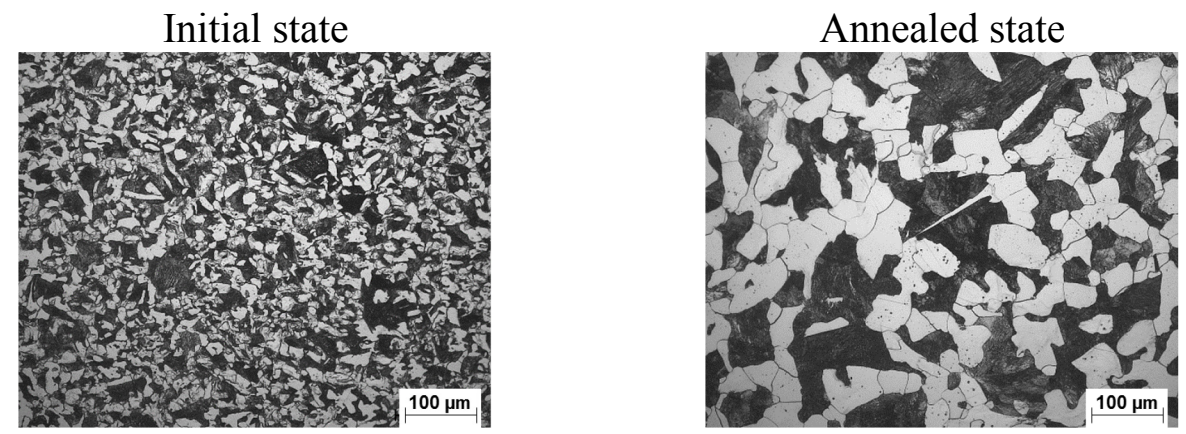

Fig. 2. Micrographs of initial and annealed state of C35

In order to describe the material behavior within the numerical simulation, the yield curves of C35 (initial state and annealed) were determined using the conventional compression test. The compression tests are performed with strain rates of $0.1 \mathrm{~s}^{-1}, 1.0 \mathrm{~s}^{-1}$ and $10.0 \mathrm{~s}^{-1}$. Based on the measured force displacement curves, a calculation of the correlating yield curves was realized using a method with correction of the friction based on Pöhlandt [7]. Fig. 3 shows the yield curve at room temperature and a strain rate of $10 \mathrm{~s}^{-1}$.

Furthermore, a precise determination of the temperature dependent specific heat capacity is necessary in order to calculate the Taylor-Quinney coefficient $\beta$ as accurate as possible. Within this study, thermogravimetry and dynamic differential calorimetry (TGA-DSC-analysis) were used simultaneously in order to determine the specific heat capacity. Fig. 4 shows the resulting curves of the specific heat capacity dependence on the temperature of the steel C35. 


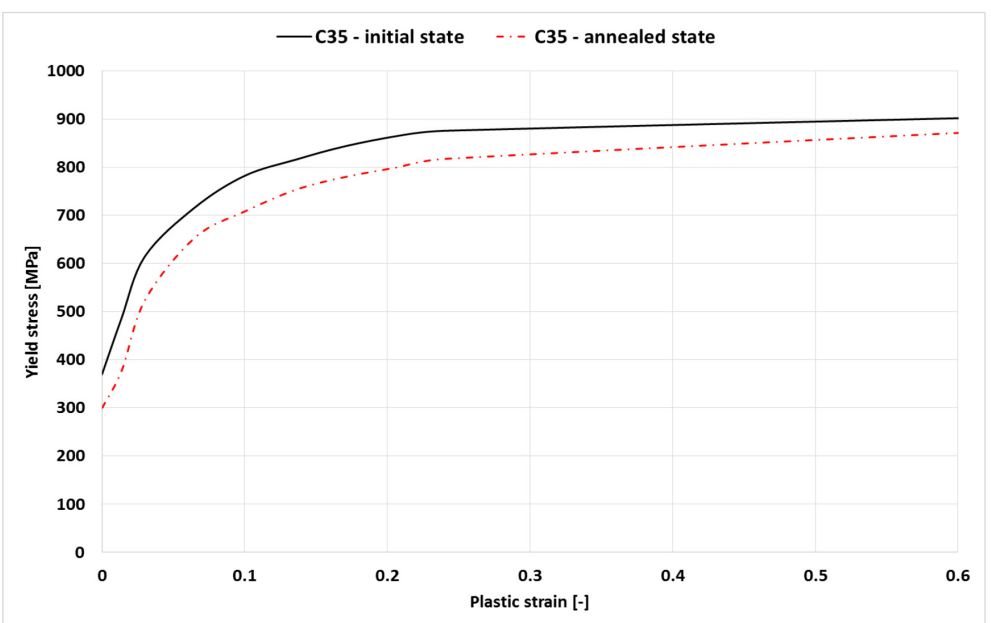

Fig. 3. Yield curve of $\mathrm{C} 35$ at room temperature and strain rate of $10 \mathrm{~s}^{-1}$

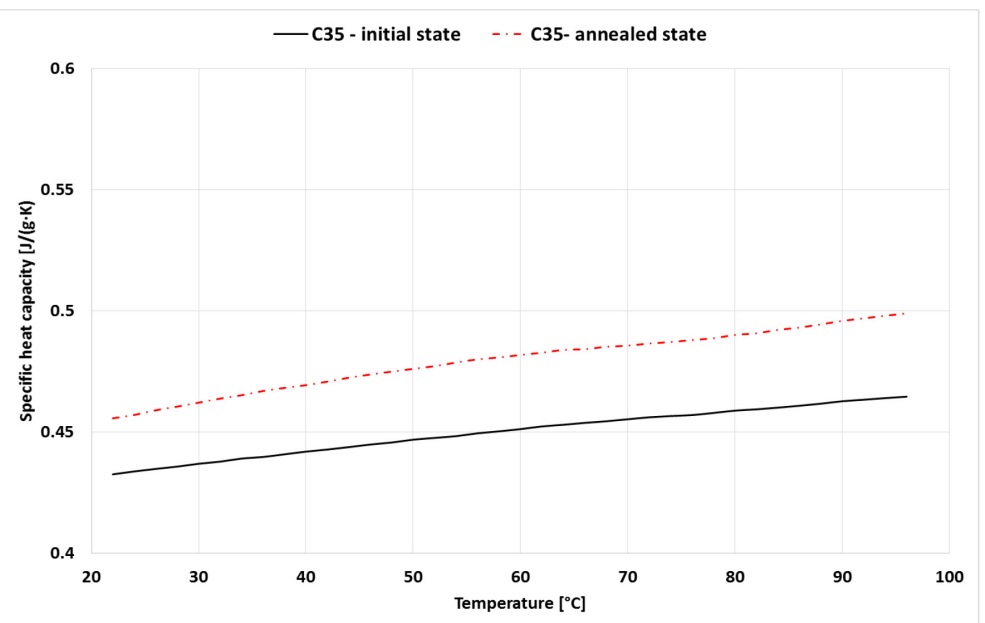

Fig. 4. Specific heat capacity depending on the temperature

\section{Numerical Determination of Suitable Specimen Geometry}

For the determination of the Taylor-Quinney coefficient $\beta$ concerning to Eq. 1, a suitable specimen geometry for the material C35 has to be developed. Therefore the temperature change in measuring point $\Delta \mathrm{T}_{\text {local }}$ has to be equal to the average temperature change of the specimen $\Delta \mathrm{T}_{\text {global }}$.

Using an inverse numerical determination method, the main dimension of the specimen are varied iteratively until the predefined Taylor-Quinney coefficient $\beta_{\text {set }}$ is nearly equal to the numerical calculated coefficient $\beta_{\text {actual }}$. Within the numerical model, the Taylor-Quinney coefficient $\beta_{\text {set}}$, the specific heat capacity $c_{p}$ and the mass $m$ are predefined as constant material parameters. The result of the numerical simulation of the upsetting process is the local change in temperature $\Delta \mathrm{T}_{\text {local }}$ and also the necessary plastic work $\mathrm{W}_{\text {plast }}$. So all values are known for calculating the TaylorQuinney coefficient $\beta_{\text {set }}$ according to Eq. 3 .

$$
\beta_{\text {actual }}=\frac{c_{\mathrm{p}} \cdot \mathrm{m} \cdot \Delta \mathrm{T}_{\text {local }}}{\mathrm{W}_{\text {plast }}}
$$

In the event that the deviation between calculated Taylor-Quinney coefficient $\beta_{\text {actual }}$ and the predefined Taylor-Quinney coefficient $\beta_{\text {set }}$ is less than $5 \%$, the specimen geometry is considered suitable. Otherwise, the diameter $D_{\text {meas }}$ and the length $L_{\text {meas }}$ of the measuring area (see Fig. 1) are varied until the permitted deviation between the calculated and predefined is achieved. The variation of diameter $D_{\text {meas }}$ and length $L_{\text {meas }}$ was done for each in the range of $3 \mathrm{~mm}-6 \mathrm{~mm}$ within steps of $0.5 \mathrm{~mm}$. The radius $\mathrm{R}$ between the supporting area and the measuring area is not varied and set to $0.5 \mathrm{~mm}$. The comparison between calculated Taylor-Quinney coefficient $\beta_{\text {actual }}$ and the predefined Taylor-Quinney coefficient $\beta_{\text {set }}$ is done for a punch stroke of $1.0 \mathrm{~mm}$. For this, the average plastic strain $\bar{\varphi}$ can be calculated for every geometry according to Eq. 4 . 


$$
\bar{\varphi}=\frac{D_{\text {meas }}}{D_{\text {meas }}-1 \mathrm{~mm}}
$$

For the inverse numerical determination of the suitable specimen geometry, a two-dimensional model is suitable because of the rotational symmetry. The workpiece is fixed between punch and bearing and the upsetting process is done with a constant punch velocity of $30 \mathrm{~mm} / \mathrm{s}$. Fig. 5 shows the numerical set-up of the rotational two-dimensional model for the determination of suitable specimen geometry (left) and the distribution of the plastic strain after upsetting of $1 \mathrm{~mm}$ (right).

The experimentally determined yield curve (see Fig. 3) as well as the specific heat capacity of C35 - initial state depending on the temperature (see Fig. 4) is used for the numerical simulation.
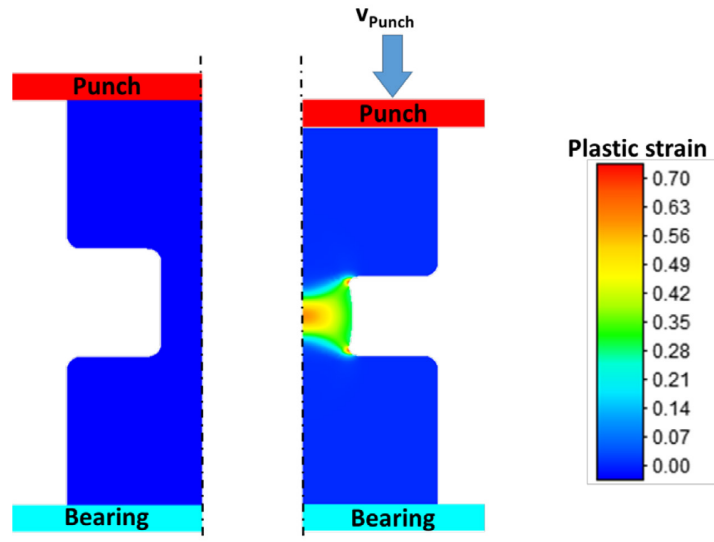

Fig. 5. Simulation setup for determination of a suitable specimen geometry and plastic strain after upsetting

Table 1 shows an extract of the analyzed specimen geometry, the average plastic strain $\bar{\varphi}$ as well as the deviation between set and actual values of the Taylor-Quinney coefficient for C35 - initial state. The numerical simulation of the upsetting process shows that the deviation between calculated and predefined Taylor-Quinney coefficient can be influenced by adapting the specimen geometry. Using geometry 3, the deviation between calculated and predefined Taylor-Quinney coefficient is $1.11 \%$. Subsequently, the local measurement of the temperature is suitable for the determination of the Taylor-Quinney coefficient for this geometry/material combination. Concerning this, geometry 3 is used for the following experimental determination of the Taylor-Quinney coefficient.

Table 1. Specimen geometry and deviation between set and actual values of Taylor-Quinney coefficient for C35 - initial state

\begin{tabular}{|l|l|l|l|l|l|l|c|}
\hline Geometry & $\mathbf{D}_{\text {meas }}$ & $\mathbf{L}_{\text {meas }}$ & $\overline{\boldsymbol{\varphi}}$ & $\boldsymbol{\beta}_{\text {set }}$ & $\boldsymbol{\beta}_{\text {actual }}$ & Deviation & Suitability \\
\hline Geometry 1 & $5.00 \mathrm{~mm}$ & $3.00 \mathrm{~mm}$ & 0.40 & 0.90 & 0.51 & $43.33 \%$ & $\times$ \\
\hline Geometry 2 & $3.00 \mathrm{~mm}$ & $3.00 \mathrm{~mm}$ & 0.40 & 0.90 & 0.70 & $22.22 \%$ & $\times$ \\
\hline Geometry 3 & $3.00 \mathrm{~mm}$ & $4.00 \mathrm{~mm}$ & 0.29 & 0.90 & 0.89 & $1.11 \%$ & $\checkmark$ \\
\hline
\end{tabular}

To ensure that geometry 3 is suitable for the Taylor-Quinney coefficient in a range between 0.7 and 1.00, additional simulations were done with set Taylor-Quinney coefficients of $0.70 ; 0.80$ and 1.00. The maximum deviation is $3.63 \%$ for a set Taylor-Quinney coefficients of 1.00 . Using the experimentally determined yield curve as well as the specific heat capacity of C35 - annealed state, the maximum deviation is also lower than $5 \%$ between calculated and predefined Taylor-Quinney coefficient.

\section{Experimental Investigations}

The determination of the Taylor-Quinney coefficient is carried out by using the thermomechanical test unit GLEEBLE 3800 (see Fig. 6, left) for upsetting tests of the novel specimen geometry. The conceptual experimental setup including measured values is shown in Fig. 6 (right). 


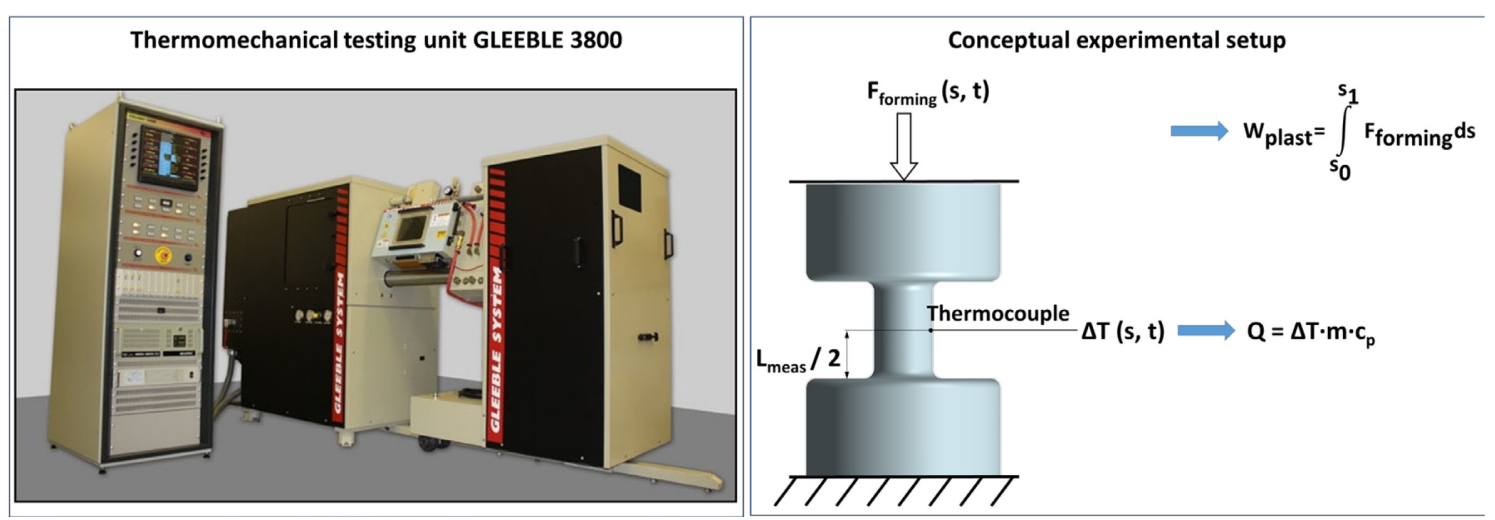

Fig. 6. GLEEBLE 3800 (le) and conceptual experimental setup including measured values (ri)

Based on the measured values and the known mass $\mathrm{m}$ of the workpiece $(0.23 \mathrm{~g}$ for steel materials), the Taylor-Quinney coefficient can be calculated using Eq. 3. For the different material states, the resulting Taylor-Quinney coefficient is given in Table 2.

Table 2. Experimental determined Taylor-Quinney coefficient for initial and annealed state of C35

\begin{tabular}{|l|l|}
\hline \multicolumn{1}{|c|}{ Material state } & Taylor-Quinney coefficient $\boldsymbol{\beta}$ \\
\hline Initial state & 0.729 \\
\hline Annealed state & 0.768 \\
\hline
\end{tabular}

The experimental investigations show that the Taylor-Quinney coefficient for the material C35 is lower than 0.9 - like generally expected. Furthermore, there is an influence of the material state. An annealing process before upsetting leads to a higher Taylor-Quinney coefficient with $\beta_{\text {annealed }}=0.768$ compared to the initial state with $\beta_{\text {initial }}=0.729$. However, it should be noted that the values are valid for an average plastic strain of $\bar{\varphi}=0.29$ and a strain rate of $\dot{\varphi}=8.70 \mathrm{~s}^{-1}$.

\section{Summary}

In this publication, a method for the determination of the Taylor-Quinney coefficient is presented. An especially developed specimen geometry allows upsetting without heating caused by friction between workpiece and tools. Furthermore, the specimen geometry is adapted iteratively until the local change in temperature is nearly equal to the global change in temperature. This was done by using the numerical simulation. Subsequently, the Taylor-Quinney coefficient was determined by upsetting the specimens. For the material C35, the Taylor-Quinney coefficient is $\beta_{\text {initial }}=0.729$ for the initial material state and $\beta_{\text {annealed }}=0.768$ for the material with a previous annealing. Therefore, an influence of the pretreatment was detected on the one hand. On the other hand, it was found that the Taylor-Quinney coefficient for the material is lower than 0.9 like generally expected.

\section{References}

[1] E. Doege, B.-A. Behrens, Handbuch Umformtechnik, second ed., Springer, Berlin; Heidelberg, 2010. ISBN 978-3-642-04248-5

[2] Taylor, G., Quinney, H., The latent heat remaining in a metal after cold working. Proceedings of the Royal Society of London A 163 (1937) 157-181.

[3] D. Ghosh, O. T. Kingstedt, G. Ravichandran, Plastic Work to Heat Conversion During HighStrain Rate Deformation of Mg and Mg Alloy, Metallurgical and Materials Transactions A Volume 48 (2017) 14-19

[4] P. Rosakis, A.J. Rosakis, G. Ravichandran, J. Hodowany, A thermodynamic internal variable model for the partition of plastic work into heat and stored energy in metals, Journal of the Mechanics and Physics of Solids 48 (2000) 581-607 
[5] P. Knysh, Y.P. Korkolis, Determination of the fraction of plastic work converted into heat in metals, Mechanics of Materials 86 (2015) 71-80

[6] B. Fekete, A. Szekeres, Investigation on partition of plastic work converted to heat during plastic deformation for reactor steels based on inverse experimental-computational method. European Journal of Mechanics A/Solids 53 (2015) 175-186

[7] K. Pöhlandt, H.-J. Becker, N. Becker, Werkstoffe und Werkstoffprüfung für die Kaltmassivumformung, first ed., .Expert-Verlag GmbH, 1994. ISBN: 978-3816909187

[8] R. Kopp, H. Wiegels, Einführung in die Umformtechnik. first ed., Verlag der Augustinus Buchhandlung, 1998. 